\title{
Resistance of two-dimensional superconducting films
}

\author{
E. J. König $\odot,{ }^{1}$ I. V. Protopopov, ${ }^{2,3}$ A. Levchenko, ${ }^{4}$ I. V. Gornyi $\odot,{ }^{5,6,7}$ and A. D. Mirlin ${ }^{5,6,3,8}$ \\ ${ }^{1}$ Max Planck Institute for Solid State Research, D-70569 Stuttgart, Germany \\ ${ }^{2}$ Department of Theoretical Physics, University of Geneva, 1211 Geneva, Switzerland \\ ${ }^{3}$ L. D. Landau Institute for Theoretical Physics RAS, 119334 Moscow, Russia \\ ${ }^{4}$ Department of Physics, University of Wisconsin-Madison, Madison, Wisconsin 53706, USA \\ ${ }^{5}$ Institute for Quantum Materials and Technologies, Karlsruhe Institute of Technology, 76021 Karlsruhe, Germany \\ ${ }^{6}$ Institut für Theorie der kondensierten Materie, Karlsruhe Institute of Technology, 76128 Karlsruhe, Germany \\ ${ }^{7}$ Ioffe Institute, 194021 St. Petersburg, Russia \\ ${ }^{8}$ Petersburg Nuclear Physics Institute, 188300 St. Petersburg, Russia
}

(Received 27 June 2021; revised 8 September 2021; accepted 9 September 2021; published 29 September 2021)

\begin{abstract}
We consider the problem of finite resistance $R$ in superconducting films with geometry of a strip of width $W$ near zero temperature. The resistance is generated by vortex configurations of the phase field. In the first type of process, quantum phase slip, the vortex world line in $2+1$ dimensional space-time is spacelike (i.e., the superconducting phase winds in time and space). In the second type, vortex tunneling, the world line is timelike (i.e., the phase winds in the two spatial directions) and connects opposite edges of the film. For moderately disordered samples, processes of the second type favor a train of vortices, each of which tunnels only across a fraction of the sample. Optimization with respect to the number of vortices yields a tunneling distance of the order of the coherence length $\xi$, and the train of vortices becomes equivalent to a quantum phase slip. Based on this theory, we find the resistance $\ln R \sim-g W / \xi$, where $g$ is the dimensionless normal-state conductance. Incorporation of quantum fluctuations indicates a quantum phase transition to an insulating state for $g \lesssim 1$.
\end{abstract}

DOI: 10.1103/PhysRevB.104.L100507

\section{INTRODUCTION}

Since its original discovery by H. Kamerlingh Onnes, a defining feature of superconductivity is its vanishing electrical resistivity in the thermodynamic limit. Yet, experimental samples are finite and can therefore be expected to display a nonzero, albeit small, resistance $R$. This naturally provokes questions about the resistance of superconductors [1,2]particularly in two-dimensional (2D) films, where resistivity and resistance have the same physical dimension: What is the dependence of $R$ on the system size and disorder strength? Is its scaling with increasing system size dual to the exponentially vanishing zero-temperature conductance of an insulator?

An approximate duality of this kind can be formalized [4] using the particle-vortex duality within the bosonic description of the superconductor-insulator transition (SIT) [5-7]. Such theories are optimized for samples, where Cooper pairs on superconducting granules Anderson delocalize at the SIT. In this paper, instead, we concentrate on the different experimental situation of homogeneous films for which the

Published by the American Physical Society under the terms of the Creative Commons Attribution 4.0 International license. Further distribution of this work must maintain attribution to the author(s) and the published article's title, journal citation, and DOI. Open access publication funded by the Max Planck Society. impurity-induced reduction and ultimate annulment of $T_{c}$ (as defined by the onset of a spectral gap) is well described by fermionic theories [8,9] and the study of resistivity below $T_{c}$ constitutes a separate, subsequent question: At finite temperature, but infinite system size, resistance is established by vortex proliferation above a renormalized BerezinskiiKosterlitz-Thouless temperature [10,11]. On the other hand, near zero temperature but at finite system size, the resistance as a function of size and disorder strength is unknown and is the subject of this paper. We focus on a $2 \mathrm{D}$ superconducting strip of width $W$ and length $L>W$, Fig. 1(a).

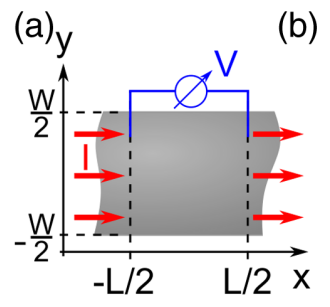

(b)

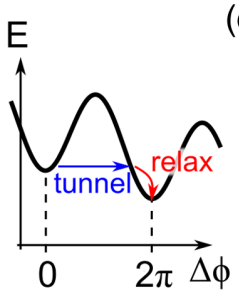

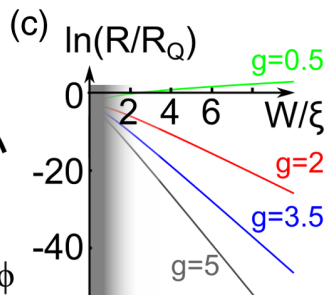

FIG. 1. (a) Setup for measuring resistance in a $2 \mathrm{D}$ superconducting film. (b) The bias current lifts the periodicity of the ground-state energy as a function of $\Delta \phi$. Tunneling events (and subsequent energy relaxation) between adjacent minima generate resistance. (c) Resistance, Eq. (8), per square (i.e., $R W / L$ ) for several values of the normal-state conductance $g$. The linear decay of $\ln R$ is consistent with experiment [3]. 
We briefly review the literature on the resistance of superconductors. Josephson's equation relates the voltage $V=$ $(d \Delta \phi / d t) \hbar / 2 e$ to the time derivative of the phase difference $\Delta \phi=\phi(x=L / 2)-\phi(x=-L / 2)$. The presence of a bias current $I$ lifts the degeneracy of quantum states with integer difference in $\Delta \phi / 2 \pi$, Fig. 1(b). Near zero temperature, quantum tunneling between adjacent minima dominates the decay of the phase difference. The voltage is then given by the effective tunneling rates $1 / \tau_{ \pm 2 \pi}$ for the change of the phase by $\pm 2 \pi$ :

$$
V=\frac{h}{2 e}\left(\frac{1}{\tau_{2 \pi}}-\frac{1}{\tau_{-2 \pi}}\right) .
$$

Theoretically, the decay of metastable vacua is described by instantonlike field configurations [12-15], i.e., imaginarytime solutions of the semiclassical equations of motion which connect the adjacent minima. For the decay of the supercurrent, the general form of such solutions is not known. However, certain trial solutions are believed to be good approximations for the present strip geometry: (i) vortex configurations which are $y$ independent and swirl in the $x-\tau$ plane ( $\tau$ is the imaginary time), i.e., the world line of the core is spacelike; and (ii) vortices which wind in the $x-y$ plane and tunnel across the system perpendicularly to the current (a timelike world line). We will refer to case (i) as quantum phase slips and (ii) as vortex tunneling.

Most of the literature on the resistance of superconductors is devoted to thermally activated decay of the supercurrent [16-18] and to vortex physics in the presence of a magnetic field [19-21] or for current bias close to the critical current. We restrict ourselves to summarizing the work in the quantum regime for quasi-1D and 2D systems at small currents and without external field. Supercurrent decay in homogeneous 1D wires was considered theoretically in Refs. [22-28]. There is a consensus that the semiclassically dominant field configuration is a dipole of phase slips at distance $\delta \tau \propto 1 / I$ in time direction, while the role of electromagnetic fields was up for debate $[23,25,29,30]$. In addition, it was shown that inhomogeneities in the wire can be crucially important $[31,32]$. For 2D samples, studies of voltage generation in ultraclean samples focus on the theory of vortex tunneling [33-36]. Quantum tunneling in the presence of inhomogeneities (pinning centers) was addressed in Refs. [33,37-39]. At the same time, the role of finite width electromagnetic gauge potentials were disregarded in these works. Further, the influence of the Magnus force and the size of the vortex mass remained controversial [40].

Experimental evidence [41] for quantum tunneling of vortices in 2D superconductors at finite current bias, in particular in the context of dark photon counts [42,43], additionally augmented interest in this research field. Modern experimental tools, such as SQUID-on-tip microscopy [44], allow accessing both vortex motion and energy dissipation (local heating). At the same time, the direct measurement of the extremely small resistance of large samples of $2 \mathrm{D}$ superconductors is experimentally challenging; most recent studies [45] demonstrate the technical subtleties and advances in the careful filtering of external radiation. A remedy is to study higher-resistance samples, e.g., not too far from the SIT or with a smaller width $W$. In the regime where a finite zero-temperature saturation value of $R$ is measurable, experimental data [3] is consistent with $-\ln R \propto W$.

In this paper, we consider moderately disordered homogeneous films with $1 / \tau_{\mathrm{el}} \gg \Delta^{2} / E_{F}$ under infinitesimal current bias. We will refer to the case $1 / \tau_{\mathrm{el}} \ll \Delta\left(1 / \tau_{\mathrm{el}} \gg \Delta\right)$ as the clean (dirty) limit. Here, $E_{F}$ is the Fermi energy, $\tau_{\mathrm{el}}$ the elastic scattering time of electrons, and $\Delta$ the spectral gap of the superconductor. As discussed below, for systems considered here, the Magnus force is unimportant and the vortex mass is finite. We find that the combined tunneling of several vortices dominates over single-vortex events and demonstrate that, for the optimal number of involved vortices, the tunneling process has the same contribution as quantum phase slips. This allows us to determine the linear-response resistance of superconducting strips, which is exponentially small but finite. Estimating the preexponential factor and pushing the theory to the border of its applicability range, we also show that the SIT due to the antagonistic interplay of energy versus entropy (here due to quantum fluctuations) is captured at sufficiently strong disorder. Beyond the strictly 2D limit, which is the focus of all calculations, our results also apply to the experimentally relevant situation of quasi-2D films of type-II superconductors, which are thinner than the correlation length.

\section{QUANTUM PHASE SLIPS}

We first consider the voltage generation by quantum phase slips. In perfectly coherent systems, the decay out of a false vacuum is technically encoded in bounce solutions: $[14,25]$ a phase slip takes the system to a lower vacuum where it dwells for a time $\delta \tau$ and subsequently an anti-phase-slip takes it back to the point of departure. The action of a bounce is $\left(\Phi_{0}\right.$ is the flux quantum)

$$
S_{I}(\delta \tau) \simeq 2 S_{\text {core }}+K_{1 \mathrm{D}} \ln (\Delta \delta \tau)-\Phi_{0} I \delta \tau .
$$

Each phase slip described by Eq. (2) is weighted by its core action $S_{\text {core }} \sim K_{1 \mathrm{D}} \sim(W / \xi) E_{F} \min \left(\tau_{\mathrm{el}}, 1 / \Delta\right)$, where the factor $(W / \xi)$ represents the length of the spacelike world line of the core. Here, we introduced the dimensionless stiffness of $1+1$ dimensional (i.e., $y$-independent) phase fluctuations $K_{1 \mathrm{D}}$ and the coherence length $\xi \sim v_{F} / \Delta\left(\xi \sim v_{F} \sqrt{\tau_{\mathrm{el}} / \Delta}\right)$ in the clean (dirty) limit, where $v_{F}$ is the Fermi velocity.

The action is maximal at the typical dwell time $\delta \tau_{\text {typ }}=$ $K_{1 \mathrm{D}} /\left(\Phi_{0} I\right)$, and a steepest-descent evaluation [46] of the instanton contribution to the partition sum [47] $\mathcal{Z}_{\text {instanton }} \sim$ $(L / \xi) \int d \delta \tau \exp \left[-S_{I}(\delta \tau)\right]$ yields the decay rate [25] 1/ $\tau_{2 \pi} \sim$ $\Delta^{2} \delta \tau_{\mathrm{typ}} \exp \left[-S_{I}\left(\delta \tau_{\mathrm{typ}}\right)\right] L /\left[\xi \sqrt{K_{1 \mathrm{D}}}\right] \sim I^{K_{\mathrm{ID}}-1}$. This nonlinear current-voltage characteristic is a manifestation of a perfectly quantum-coherent bounce. However, at infinitesimal bias current, $\delta \tau_{\text {typ }}$ exceeds the relaxation time $\tau_{\text {coh }}$ which is always finite in a finite system connected to the metallic leads or in the presence of an external bath. The broadening of levels inside the core described by $\tau_{\text {coh }}$ leads to a finite rate for quantum phase slips and anti-phase-slips $1 / \tau_{ \pm 2 \pi} \propto e^{-S_{ \pm I}\left(\tau_{\mathrm{coh}}\right)} L /\left(\tau_{\mathrm{coh}} \xi\right)$. Then, Eq. (1) leads to a linear current-voltage relation,

$$
R_{\mathrm{QPS}} \sim \frac{h}{e^{2}} \frac{L}{\xi} K_{1 \mathrm{D}}^{-3 / 2}\left(\tau_{\mathrm{coh}} \Delta\right)^{2-K_{\mathrm{ID}}} e^{-2 K_{\mathrm{ID}}},
$$


where the prefactor is determined by matching the nonlinear resistance at $I \gtrsim K_{1 \mathrm{D}} /\left(\Phi_{0} \tau_{\text {coh }}\right)$ mentioned above.

We conclude this consideration with three remarks. First, we comment on $\tau_{\text {coh }}$ which is finite in any realistic situation and may result from a variety of system-dependent origins, such as phonons, external radiation, and noise in the leads. Different sources generally imply different temperature and system-size dependence of the relaxation time which we do not study here, since the main factor in $-\ln R$ is the tunneling action, while $\tau_{\text {coh }}$ enters in the form of a logarithmic prefactor. Second, spatial fluctuation of the world line of the phase-slip core around the $y$-independent line have been disregarded up to now. These fluctuations lead to an additional preexponential factor, which we estimate at the end of the paper. Third, magnetic screening effects (see Ref. [29] and Supplemental Material [48]) are unimportant in the above consideration [30] so long as $\tau_{\text {coh }} \Delta \ll \exp \left(\lambda_{M} / W\right)$, where $\lambda_{M}$ is the Pearl length (2D analog of the London penetration length), which is usually macroscopic. For longer relaxation times, the result for $-\ln R$ gets modified according to $K_{1 \mathrm{D}} \ln \left(\tau_{\mathrm{coh}} \Delta\right) \rightarrow$ $K_{1 \mathrm{D}} \lambda_{M} \ln \left[W \ln \left(\tau_{\mathrm{coh}} \Delta\right) / \lambda_{M}\right] / W$.

\section{VORTEX DYNAMICS}

When the width of the sample exceeds the superconducting coherence length, vortices in the $x-y$ plane of the superconducting film become well-defined topological excitations and the supercurrent acquires an additional decay channel related to vortex tunneling across the sample. This effect therefore relies on the dynamics of vortices, described by the action $S_{\text {kin }}=\int d \tau\left(m \dot{x}^{2} / 2+i \alpha \dot{x} y\right)+\eta \int d \omega|\omega||x(\omega)|^{2} /(2 \pi)$, where $x(\tau)=(x(\tau), y(\tau))$ is the time-dependent position of a given vortex. The first term represents the kinetic energy for a point particle of mass $m$, the term proportional to $\alpha$ describes the Magnus force, which is somewhat similar to the Lorentz force in a magnetic field, and the last term, proportional to $\eta$, describes dissipation.

The values of these parameters drastically depend on whether the vortex core is featureless or whether it contains a quasicontinuum of bound states. In the first case, dissipation is absent $(\eta=0)$ and the parameter $\alpha$ is topologically quantized (it is given by the superfluid density) [49]. The mass diverges logarithmically if the superfluid is neutral, but the logarithm is cut inside the core for charged superconductors, where the mass is therefore minute, $m \sim m_{\text {electron }} \lambda_{\mathrm{TF}} / \xi$ (here, $\lambda_{\mathrm{TF}}$ is the Thomas-Fermi screening length) [50-54].

Here, we concentrate on the more realistic second case, in which the Caroli-deGennes-Matricon-type [55] bound states inside the vortex core cannot be neglected. In $s$-wave superconductors, the spacing $\omega_{0}$ of these subgap states [56] can be estimated to be $\omega_{0} \sim v^{-1} \xi^{-2}$, where $v$ is the metallic density of states. Typically, in the clean (dirty) limit, $\omega_{0} \sim \Delta^{2} / E_{F}$ $\left(\omega_{0} \sim \Delta / E_{F} \tau_{\mathrm{el}}\right)$ is negligible compared to $1 / \tau_{\mathrm{el}}$. For a moving vortex that explores different microscopic realizations of disorder, this leads to a quasicontinuum of states, which allows for energy dissipation, yielding $\eta \sim n \omega_{0} \tau_{\mathrm{el}}$, where $n$ is the density of electrons in the normal state [37,57]. At the same time, the Magnus force acquires a second topological contribution resulting from the spectral flow of bound states, which is equal in magnitude but opposite to the hydrodynamic

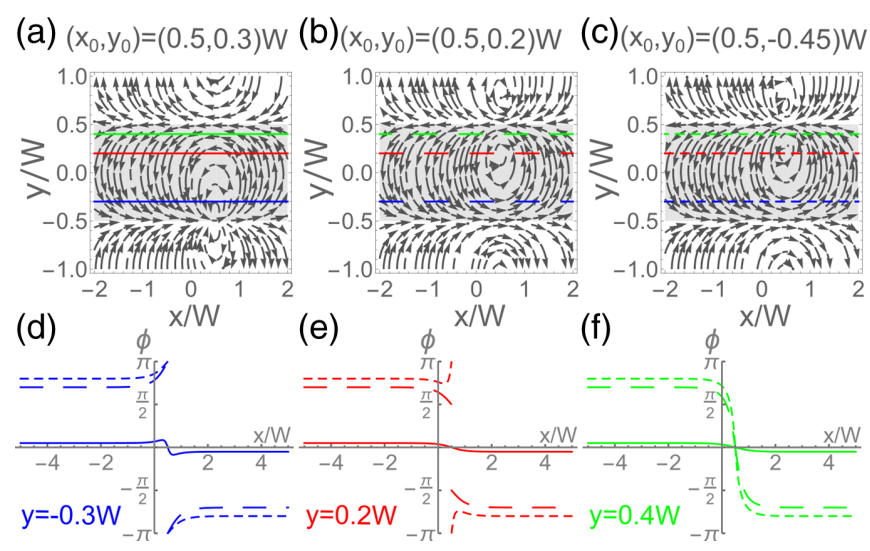

FIG. 2. A single vortex at position $\left(x_{0}, y_{0}\right)$ tunneling from lower to the upper edge. (a)-(c) Gradient of the superconducting phase $\nabla \phi$. Note that mirror charges outside the sample (gray shaded) ensure no current outflux. (d)-(f) Principal branch of the phase $\phi$ along the colored horizontal lines of panels (a)-(c) (same color and dashing code).

contribution discussed above, so $\alpha$ effectively vanishes $[58,59]$. Finally, the vortex mass is given by the total mass of particles trapped inside the vortex, $m \sim m_{\text {electron }} \xi^{2} / \lambda_{F}^{2}$ [59].

\section{SINGLE VORTEX TUNNELING}

We first inspect tunneling events involving a single vortex in the system and remind the reader of the logarithmic attraction of a vortex-antivortex pair. It is, perhaps, less known that a single vortex in a finite superconducting strip is attracted to the boundaries. Technically, this results from the implementation of no-current-outflux boundary conditions by means of mirror charges [Figs. 2(a)-2(c) and Supplemental Material [48]) and leads to a potential $V(y)=2 J \ln [W \cos (\pi y / W) / \xi]$ that should be included in the total action of the vortex in the strip geometry. Here, $J$ is the 2D superconducting stiffness: $J \sim E_{F}$ $\left(J \sim E_{F} \Delta \tau_{\mathrm{el}}\right)$ in the clean (dirty) limit at zero temperature.

We begin by considering a kink solution, $y_{\text {kink }}(\tau)$, of the equation of motion, i.e., the unbinding of a single vortex from lower boundary and subsequent tunneling across the system, Fig. 2. The tunneling action is $S_{\text {kink }}(W)=S_{\text {pot }}(W)+$ $S_{\text {cond }}(W)$, where

$$
S_{\mathrm{pot}}=\int_{-W / 2+\xi}^{W / 2-\xi} d y \sqrt{2 m V(y)}=2 W \sqrt{m J} f(\pi \xi / W),
$$

is the contribution from the potential barrier. Here, we have introduced the dimensionless function $f(x)$ that satisfies $f(x) \simeq \sqrt{\ln (1 / x)}$ in the limit $x \ll 1$ and $f(\pi / 2)=$ 0 . The second contribution, $S_{\text {cond }}=E_{\text {cond }} \mathcal{T}$, is the condensation energy $E_{\text {cond }} \sim J$ times the total length of the world line $\mathcal{T}=\mathcal{T}(W)$. Here, $\mathcal{T}(W)=W /[v \sqrt{\ln (W / \pi \xi)}]$ for wide strips $W \gg \xi$ (with $v=\sqrt{J / m} \sim v_{F} \Delta / E_{F}$ the typical vortex speed), whereas for $W \rightarrow 2 \xi, \mathcal{T}(W) \rightarrow 1 / \Delta$, which is the time for a vortex to nucleate. Thus, for narrow strips, $S_{\text {kink }}(W \rightarrow 2 \xi) \simeq J /\left.\Delta \sim K_{1 \mathrm{D}}\right|_{W \sim \xi}$ approaches the same value as the core action of a quantum phase slip, while for wide strips, both $S_{\text {pot }}(W)$ and $S_{\text {cond }}(W)$ are of the 
order of $J W / v \sim\left(W / \lambda_{F}\right) E_{F} \min \left(\tau_{\mathrm{el}}, 1 / \Delta\right)$, which is $\left(\xi / \lambda_{F}\right)$ times larger than the core action of a phase slip in Eq. (2).

A finite current bias lifts the degeneracy of the potential minima at $y= \pm W / 2$ by an additional term $V_{I}=-\Phi_{0} I y / W$. As mentioned, the supercurrent decay is governed by a bounce solution [14,47], which we approximate by a kink and an antikink at temporal distance $\delta \tau: y_{\text {bounce }}(\tau)=y_{\text {kink }}(\tau+\delta \tau / 2)-$ $y_{\text {kink }}(\tau-\delta \tau / 2)+W / 2-\xi$. Using this ansatz, we obtain the following $\delta \tau$-dependent action, see Supplemental Material [48]:

$$
S_{I}(\delta \tau)=2 S_{\text {kink }}(W)+S_{\text {int }}(\delta \tau)-\Phi_{0} I \delta \tau .
$$

At variance with the 1D bounce solution, Eq. (2), the attraction between kink and antikink, encoded in $S_{\text {int }}(\delta \tau)$, cannot be determined exactly. It asymptotically vanishes if the vortex disappears at the opposite boundary [60] $S_{\text {int }}(\delta \tau) \rightarrow 0$ for $\delta \tau>\mathcal{T}$ and monotonically increases in the preceding regime $0<\mathcal{T}-\delta \tau \ll \mathcal{T}$, where $S_{\text {int }}(\delta \tau) \simeq-E_{\text {cond }}(\mathcal{T}-\delta \tau)$, which shows that the dominant reason for attraction is the string tension of the vortex world line.

Formally, the instanton contribution to the partition sum can be evaluated using the steepest-descent method analogously to the bounce action Eq. (2). However, the properties of $S_{\text {int }}(\delta \tau)$ imply that the typical dwell time $\delta \tau$ diverges as a function of $I \rightarrow 0$, exceeding the coherence time $\tau_{\mathrm{coh}} \gg \mathcal{T}$. Consequently, evaluation of Eq. (1) similarly to Eq. (3) but using Eq. (5) instead of Eq. (2) yields

$$
R_{\mathrm{SvT}}=\frac{h}{e^{2}} \frac{L}{\xi} \mathcal{A}_{\mathrm{vortex}} e^{-2 S_{\text {kink }}(W)} .
$$

The pre-exponential factor $\mathcal{A}_{\text {vortex }}$ depends on the nature of relaxation and should be evaluated for a given microscopic model of relaxation.

We conclude the discussion of a single-vortex tunneling with three comments. First, the logarithmic vortex interaction is cut beyond the Pearl length [48] $\lambda_{M}$ so Eq. (4) is valid only for samples $W<\lambda_{M}$. Second, the effect of dissipation on the tunneling has been disregarded here, which is valid if the tunneling time $\mathcal{T}$ is small compared to the dissipation time $m / \eta \sim 1 / \omega_{0}^{2} \tau_{\mathrm{el}}$. This is equivalent to the condition $W \ll$ $\min \left(v_{F} \tau_{\mathrm{el}}, \xi\right) /\left(\omega_{0} \tau_{\mathrm{el}}\right)$. We will see in the next section that the resistance is dominated by vortices tunneling across an effective distance $d_{\mathrm{opt}} \ll W$, which satisfies both the bounds imposed by screening and by dissipation, even for wide junctions. Third, in deriving the effective tunneling action, we disregarded the mesoscopic fluctuations of the superconducting gap (and, hence, stiffness $J$ ) $[11,61]$. As we demonstrate in Ref. [48] for $E_{F} \tau_{\mathrm{el}} \gg 1$, these fluctuations do not affect the exponential factor in Eq. (6).

\section{MULTIVORTEX TUNNELING}

A simple tunneling event that involves more than one vortex is a dipole dissociation [34] in which a quantum fluctuation creates a dipole of vortices inside the strip and subsequently the dipole constituents tunnel toward opposite edges, leading to an overall phase slip of $2 \pi$. Here, we consider a generalization of this event where $n$ dipoles are nucleated in the strip and subsequently tunnel to either side of the strip, Fig. 3(b). We also consider a generalization of (a)

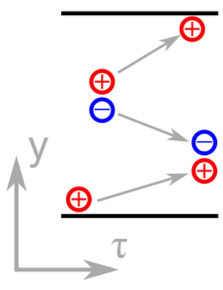

(b)

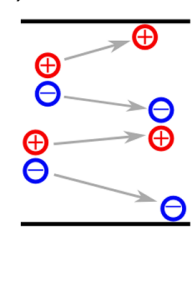

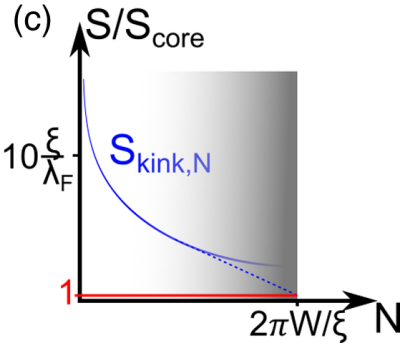

FIG. 3. (a) The unbinding of a vortex from the lower edge, similar to Fig. 2, but assisted by an additional dipole nucleating in the bulk of the system. (b) The decay of two vortex dipoles into a dipole and two vortices disappearing at the edge. (c) The tunneling action [in units of $S_{\text {core }}$ introduced in Eq. (2)] for a multivortex tunneling process as a function of $N$.

the edge unbinding event discussed above, Fig. 2, where the tunneling of a vortex from one edge to the other is assisted by $n$ dipoles in the bulk, Fig. 3(a).

As a natural ansatz, we consider [48] the total action $S_{\text {kink,N }}=\sum_{i=1}^{N} S_{\text {kink }}\left(d_{i}\right)$, where $S_{\text {kink }}(d)$ is the kink action for a single vortex tunneling in a strip of size $d$ introduced before Eq. (4), $d_{i}$ is the tunneling distance of the $i$ th vortex, and $N=2 n+1$ in the case of Fig. 3(a) and $N=2 n$ in case of Fig. 3(b). The minimization of the action prescribes that all dipoles nucleate at the same moment in time and same $x$ position, and that the nucleation points are furthermore equally spaced in the $y$ direction, i.e., each vortex travels the same distance $d_{N}=W / N$, so the kink action is $S_{\text {kink, N }}=N S_{\text {kink }}\left(d_{N}\right)$. We remark that this is an accurate tunneling action for the optimum event; yet, the ansatz that we used is heuristic and employing it for the calculation of fluctuation determinant is not exact, although parametrically correct.

Increasing $N$ in $S_{\text {kink, }}$ has two effects. On one hand, $N S_{\text {pot }}\left(d_{N}\right)$ decreases with the number of dipoles. On the other hand, the contribution of the string tension $N E_{\text {cond }} \mathcal{T}\left(d_{N}\right)$ increases with $N$ [even though $\mathcal{T}\left(d_{N}\right)$, of course, decreases]. The first effect prevails, Fig. 3(c), in which the solid curve is obtained on the basis of the vortex motion at length scales large compared to $\xi$, and the dashed curve is the extrapolation of $\mathcal{T}\left(d_{N}\right) \searrow 1 / \Delta$ as $d_{N}$ approaches $\xi$ (shaded region). The action monotonically decreases with an increasing number of vortices, leading to an optimum number $N_{\text {opt }} \sim W / \xi$ and $d_{N} \sim 2 \xi$. The resistance produced by multivortex configurations,

$$
R_{\mathrm{MVT}}=\frac{h}{e^{2}} \frac{L}{\xi} \mathcal{A} e^{-2(W / \xi) S_{\text {kink }}(2 \xi)},
$$

follows along the lines of the calculation for the single-vortex tunneling event (the pre-exponential factor $\mathcal{A}$, which may depend on relaxation details, is considered separately). Clearly, as $d_{N} \sim \xi$, vortices cease being well-defined excitations, and the multivortex tunneling event cannot be distinguished from a quantum phase slip. It is, therefore, a reassuring consistency check that the exponent in the resistance due to $N$ cotunneling vortices, Eq. (7), $N S_{\text {kink }}(2 \xi) \sim N J / \Delta \sim$ $W E_{F} \min \left(1 / \Delta, \tau_{\mathrm{el}}\right) / \xi \sim K_{1 \mathrm{D}}$, is in accordance with the exponent of the resistance due to quantum phase slips, Eq. (3). 


\section{CONCLUSION}

In summary, we have investigated the resistance of superconducting strips with moderate disorder near zero temperature. The obtained resistance $R$ is dominated by cotunneling of $N \sim W / \xi>1$ vortices, a process that is equivalent to a quasi-1D quantum phase slip, see Eqs. (3) and (7). These results also apply to quasi-2D films with thickness $d_{z} \in\left(\lambda_{F}, \xi\right)$, then the parameters $K_{1 \mathrm{D}}, S_{\text {kink }}$ contain additional factors [48] of $d_{z} / \lambda_{F}$. The exponential dependence of resistance on $W$ is reminiscent of exponential suppression of conductance with the system size on the insulating side of SIT.

While we concentrated on the exponent of the superconducting decay rate, we conclude with a discussion of the preexponential factor. Its $W$ dependence stems from quantum fluctuations of the tunneling trajectory (if dissipation is local). Using the above-mentioned ansatz for multivortex events, we estimate that each of the $W / \xi$ vortices contributes to $\mathcal{A}$ in Eq. (7), a fluctuation determinant of the order of the inverse kink action [48], leading to a pre-exponential factor $\left[S_{\text {kink }}(2 \xi)\right]^{-W / \xi}$. A similar factor is expected to arise from spatial fluctuations about the straight $y$-independent phase-slip world line. By re-exponentiation, this quantum-fluctuation- induced prefactor can be viewed as an entropic contribution to the tunneling action, leading to

$$
\ln \left(e^{2} R / h\right) \sim-W[g+\ln (g)] / \xi+\ln (L / \xi)
$$

in the dirty limit, which qualitatively agrees with the low- $T$ saturation values of resistance presented in Fig. 1 of Ref. [3], see Fig. 1(c). In Eq. (8), $g=E_{F} \tau_{\mathrm{el}}\left(g=E_{F} \tau_{\mathrm{el}} d_{z} / \lambda_{F}\right)$ is the normal-state conductance in the 2D limit (quasi-2D film). Pushing our theory to the border of its applicability, we observe a sign change of the action at $g \sim 1$, reminiscent of SIT.

\section{ACKNOWLEDGMENTS}

We acknowledge useful discussions with D. Bagrets, A. Bezryadin, I. Burmistrov, A. Finkelstein, E. Khalaf, A. Rogachev, D. Shahar, M. Skvortsov, C. Strunk, H. Xie, and A. Zaikin. Support for this work at the University of Wisconsin-Madison (A.L.) was provided by the National Science Foundation, Quantum Leap Challenge Institute for Hybrid Quantum Architectures and Networks, NSF Grant No. 2016136.
[1] D. A. Huse, M. P. Fisher, and D. S. Fisher, Nature (London) 358, 553 (1992).

[2] B. I. Halperin, G. Refael, and E. Demler, in BCS: 50 Years, edited by L. Cooper and D. Feldman (World Scientific, Singapore, 2011).

[3] I. Schneider, K. Kronfeldner, T. I. Baturina, and C. Strunk, Phys. Rev. B 99, 094522 (2019).

[4] M. P. A. Fisher, Phys. Rev. Lett. 65, 923 (1990).

[5] V. F. Gantmakher and V. T. Dolgopolov, Phys. Usp. 53, 1 (2010).

[6] Y.-h. Lin, J. Nelson, and A. M. Goldman, Phys. C 514, 130 (2015).

[7] B. Sacépé, M. Feigel'man, and T. M. Klapwijk, Nat. Phys. 16, 734 (2020).

[8] A. Finkelstein, Sov. Sci. Rev., Sect. A 14, 1 (1990).

[9] I. S. Burmistrov, I. V. Gornyi, and A. D. Mirlin, Phys. Rev. B 92, 014506 (2015).

[10] B. Halperin and D. Nelson, J. Low Temp. Phys. 36, 599 (1979).

[11] E. J. König, A. Levchenko, I. V. Protopopov, I. V. Gornyi, I. S. Burmistrov, and A. D. Mirlin, Phys. Rev. B 92, 214503 (2015).

[12] A. Vainshtein, Decaying systems and divergence of the series of perturbation theory (unpublished).

[13] J. Langer, Ann. Phys. 41, 108 (1967).

[14] S. Coleman, Phys. Rev. D 15, 2929 (1977).

[15] C. G. Callan and S. Coleman, Phys. Rev. D 16, 1762 (1977).

[16] J. Langer and V. Ambegaokar, Phys. Rev. 164, 498 (1967).

[17] D. E. McCumber and B. I. Halperin, Phys. Rev. B 1, 1054 (1970).

[18] Y. N. Ovchinnikov and A. A. Varlamov, Phys. Rev. B 91, 014514 (2015).

[19] P. W. Anderson, Phys. Rev. Lett. 9, 309 (1962).

[20] G. Blatter, V. B. Geshkenbein, and V. M. Vinokur, Phys. Rev. Lett. 66, 3297 (1991).
[21] G. Blatter, M. V. Feigel'man, V. B. Geshkenbein, A. I. Larkin, and V. M. Vinokur, Rev. Mod. Phys. 66, 1125 (1994).

[22] S. Saito and Y. Murayama, Phys. Lett. A 139, 85 (1989).

[23] J.-M. Duan, Phys. Rev. Lett. 74, 5128 (1995).

[24] S. R. Renn and J.-M. Duan, Phys. Rev. Lett. 76, 3400 (1996).

[25] A. D. Zaikin, D. S. Golubev, A. van Otterlo, and G. T. Zimányi, Phys. Rev. Lett. 78, 1552 (1997).

[26] D. S. Golubev and A. D. Zaikin, Phys. Rev. B 64, 014504 (2001).

[27] S. Khlebnikov, Phys. Rev. Lett. 93, 090403 (2004).

[28] G. Refael, E. Demler, Y. Oreg, and D. S. Fisher, Phys. Rev. B 75, 014522 (2007).

[29] J.-M. Duan, Phys. Rev. Lett. 79, 3316 (1997).

[30] A. D. Zaikin, D. S. Golubev, A. van Otterlo, and G. T. Zimányi, Phys. Rev. Lett. 79, 3317 (1997).

[31] G. V. Pai, E. Shimshoni, and N. Andrei, Phys. Rev. B 77, 104528 (2008).

[32] M. Vanević and Y. V. Nazarov, Phys. Rev. Lett. 108, 187002 (2012).

[33] P. Ao and D. J. Thouless, Phys. Rev. Lett. 72, 132 (1994).

[34] R. Iengo and G. Jug, Phys. Rev. B 52, 7537 (1995).

[35] R. Iengo and G. Jug, Phys. Rev. B 54, 13207 (1996).

[36] D. P. Arovas and A. Auerbach, Phys. Rev. B 78, 094508 (2008).

[37] M. Feigel'man, V. Geshkenbein, A. Larkin, and S. Levit, JETP Lett. 57, 711 (1993).

[38] M. J. Stephen, Phys. Rev. Lett. 72, 1534 (1994).

[39] A. Auerbach, D. P. Arovas, and S. Ghosh, Phys. Rev. B 74, 064511 (2006).

[40] D. J. Thouless, P. Ao, Q. Niu, M. R. Geller, and C. Wexler, Int. J. Mod. Phys. B 13, 675 (1999).

[41] F. Tafuri, J. R. Kirtley, D. Born, D. Stornaiuolo, P. G. Medaglia, P. Orgiani, G. Balestrino, and V. G. Kogan, Europhys. Lett. 73, 948 (2006). 
[42] J. Kitaygorsky et al., IEEE Trans. Appl. Supercond. 17, 275 (2007).

[43] L. N. Bulaevskii, M. J. Graf, C. D. Batista, and V. G. Kogan, Phys. Rev. B 83, 144526 (2011).

[44] L. Embon, Y. Anahory, Z. L. Jelić, E. O. Lachman, Y. Myasoedov, M. E. Huber, G. P. Mikitik, A. V. Silhanek, M. V. Milosević, A. Gurevich, et al., Nat. Commun. 8, 1 (2017).

[45] I. Tamir, A. Benyamini, E. Telford, F. Gorniaczyk, A. Doron, T. Levinson, D. Wang, F. Gay, B. Sacépé, J. Hone, et al., Sci. Adv. 5, eaau3826 (2019).

[46] Reference [25] evaluated the integral $\int d \delta \tau \exp [-S(\delta \tau)]$ exactly and found an exponent $R \sim I^{K_{1 \mathrm{D}}-2}$. It is in agreement with the result $R \sim I^{K_{1 \mathrm{D}}-1}$ of the steepest-descent method used in our work in its regime of applicability, $K_{1 \mathrm{D}} \gg 1$. The advantage of our approach is that it is applicable for both quantum phase slips and $2 \mathrm{D}$ vortex tunneling.

[47] H. Kleinert, Path Integrals in Quantum Mechanics, Statistics, Polymer Physics, and Financial Markets, EBL-Schweitzer (World Scientific, Singapore, 2009).

[48] See Supplemental Material at http://link.aps.org/supplemental/ 10.1103/PhysRevB.104.L100507 for an overview list of scales, a derivation of the vortex interaction in the strip, as well as details about vortex tunneling, multivortex configurations and mesoscopic fluctuations, including Refs. [62,63].

[49] P. Ao and D. J. Thouless, Phys. Rev. Lett. 70, 2158 (1993).
[50] H. Suhl, Phys. Rev. Lett. 14, 226 (1965).

[51] E. Brandt, J. Low Temp. Phys. 26, 735 (1977).

[52] J.-M. Duan and A. J. Leggett, Phys. Rev. Lett. 68, 1216 (1992).

[53] J.-M. Duan, Phys. Rev. B 48, 333 (1993).

[54] E. Demircan, P. Ao, and Q. Niu, Phys. Rev. B 54, 10027 (1996).

[55] C. Caroli, P. De Gennes, and J. Matricon, Phys. Lett. 9, 307 (1964).

[56] In what follows, a possible zero-bias anomaly structure in the density of the core bound states (peak or dip, depending on the strength of disorder $[55,64]$ ) will be inessential for our analysis.

[57] J. Bardeen and M. J. Stephen, Phys. Rev. 140, A1197 (1965).

[58] N. Kopnin and V. Kravtsov, JETP Lett. 23, 578 (1976).

[59] G. E. Volovik, The Universe in a Helium Droplet (Oxford University Press on Demand, 2003), Vol. 117.

[60] Here, we do not describe microscopically the process of vortex disappearance at the boundary, assuming that the relaxation described by $\tau_{\text {coh }}$ is sufficient for transferring the core electrons to the condensate or to the leads.

[61] M. A. Skvortsov and M. V. Feigel'man, Phys. Rev. Lett. 95, 057002 (2005).

[62] E. Abrahams and T. Tsuneto, Phys. Rev. 152, 416 (1966).

[63] H. T. C. Stoof, Phys. Rev. B 47, 7979 (1993).

[64] A. Datta, A. Banerjee, N. Trivedi, and A. Ghosal, New paradigm for a disordered superconductor in a magnetic field, arXiv:2101.00220. 